\title{
Effects of castration on the immunoreactivity to NGF, BDNF and their receptors in the pelvic ganglia of the male rat
}

\author{
C. Squillacioti, A. De Luca, G. Paino, N. Mirabella \\ Department of Structures, Functions and Biological Technologies, University of Naples Federico II, \\ Napoli, Italy
}

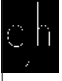

(C)2008 European Journal of Histochemistry

Nerve growth factor (NGF) and brain derived neurotrophic factor (BDNF) and are members of the neurotrophin family, a family of neurotrophic factors that also includes neurotrophin (NT) 3 and NT4/5. Neurotrophins have essential roles in the survival, development and differentiation of neurons in the central and peripheral nervous systems. Neurotrophins exert their effects by binding to corresponding receptors which are formed by the tyrosine protein kinases TrkA, TrkB and TrkC, and the low affinity neurotrophic receptor (p75 $\left.5^{\mathrm{NR}}\right)$.

In the present study, using immunohistochemistry and quantitative analysis, we have investigated immunoreactivity to BDNF, NGF, TrkB, p75 $5^{\mathrm{NTR}}$ and TrkA in the pelvic ganglia of normal and castrated rats. Neurons of the pelvic ganglia expressed both these neurotrophins and their receptors. After castration the immunoreactivity persisted. However, the number of BDNF- and $p 75^{\mathrm{NTR}}$-IR cells statistically significant decreased after castration. These results suggest that castration modulates the expression of neurotrophins and their receptors in pelvic autonomic neurons.

Key words: Trks, p75 ${ }^{\mathrm{NTR}}$, immunohistochemistry, pelvic ganglia.

Correspondence: Caterina Squillacioti,

Department of Structures,

Functions and Biological Technologies,

University of Naples Federico II,

Via Veterinaria 1, 80137, Napoli, Italy

Tel.: +39.081.2536113.

Fax: +39.081 .2536097$

E-mail: caterina.squillacioti@unina.it

Paper accepted on May 12, 2008

European Journal of Histochemistry

2008; vol. 52 issue 2 (Apr-Jun): 101-106
$\mathrm{N}$ erve growth factor (NGF) and brain derived neurotrophic factor (BDNF) are members of the neurotrophin family, a family of neurotrophic factors that also includes neurotrophin (NT) 3 and NT4/5. Neurotrophins have essential roles in the survival, development and differentiation of neurons in the central and peripheral nervous systems (Levi-Montalcini, 1987; Ernfors et al. 1994; Snider 1994; Huang and Reichart 2001; Murer et al. 2001). In addition to their effects in neural survival and differentiation, neurotrophins are now implicated in neurite outgrowth, synaptic plasticity, cell cycle control and apoptosis during adulthood (Schinder and Poo, 2000; Chao, 2003). Furthermore, recent data show that neurotrophins are involved in a variety of biological processes in nonneuronal tissues in humans and other vertebrates (Yamamoto et al., 1996; Sariola, 2001; Leon et al. 1994; Rosenbaum et al. 1998; Tessarollo, 1998).

The functions of neurotrophins are mediated through their binding to two distinct types of cell surface receptors, the receptors of tropomyosinerelated kinase family tyrosine kinases (TrkA, TrkB and TrkC) (Barbacid 1995; Lewin and Barde 1996; Patapoutian and Reichart 2001) and the panneurotrophinreceptor p75 ( $p 75^{\mathrm{NTR}}$ ), a member of the tumor necrosis factor (TNF) receptor family (Teng and Hempstead, 2004). The Trk receptors are specific for their ligands; NGF is the preferred ligand for TrkA, BDNF and NT-4/5 are preferred ligands for TrkB and NT-3 is the preferred ligand for TrkC.

Pelvic ganglia are clusters of autonomic neurons that are final motor pathways mediating storage and voiding functions of the lower urinary tract and bowel, and reproductive behaviours. They comprise a mixture of sympathetic and parasympathetic neurons. The male rat possesses large, paired major pelvic ganglia (MPG) located close to the dorsal surface of the prostate gland. Nearby lies a cluster 
of very small ganglia, the accessory ganglia, which are considered to be functionally identical to the major pelvic ganglia, except that they provide little innervation of non-reproductive targets (Purinton et al., 1973; for a review, Keast JR, 2006).

Adult rat noradrenergic pelvic ganglion neurons express the neurotrophin receptors, TrkA and p75 ${ }^{\text {NTR }}$ (Keast and Kepper, 2001), as would be expected for NGF-sensitive neurons (Huang and Reichart, 2001). In addition, it has been observed that NGF, BDNF and TrkB were expressed in major pelvic ganglion neurons and their expression increased after cyclophosphamide-induced cystitis in rats (Murray et al. 2004).

Pelvic ganglia show a remarkable sensitivity to circulating androgens in adult animals as they undergo a marked reduction in soma size and enzyme content under conditions of androgen deficiency (Keast and Saunders 1998; Keast, 2000; Keast et al. 2002; Mirabella et al. 2003). Recent studies suggest that the expression of BDNF, TrkB and $p 75^{\mathrm{NTR}}$ in the vas deferens and accessory male genital glands of the rat is influenced by androgen hormones (Mirabella et al. 2006; Mirabella et al., 2007).

The aim of the present study was to investigate, by using immunohistochemistry and quantitative analysis, the distribution of NGF, BDNF, TrkB, p75 ${ }^{\mathrm{NTR}}$ and TrkA in the pelvic ganglia and, most importantly, how this distribution can be influenced by castration. This could provide important information about the role of neurotrophins in regulating castration-induced involutive processes occurring in these ganglia.

\section{Materials and Methods}

A total of 10 adult male Sprague-Dawley rats (13 weeks of age; body weight: $380 \pm 20$ g; purchased from Harlan Italy) were used. They were housed in temperature- and light-controlled rooms and were given water ad libitum. Rats received humane care and the study protocol was in compliance with our institution's ethical guidelines. All procedures were approved by Italian laws regarding animal use in research (art. 7 D.Lgs. 116/92). Five rats were left intact and five rats were castrated. The rats were castrated at 9 weeks of age (body weight: $300 \pm 20 \mathrm{~g}$ ) and allowed to recover over the following 4 weeks. All surgical procedures were carried out aseptically under anaesthesia induced with urethane $(1.2 \mathrm{~g} / \mathrm{kg})$. Pelvic ganglia were removed from normal and castrated rats after they had been anaesthetized and then killed.

\section{Immunohistochemistry}

The specimens were immediately fixed by immersion in Bouin's fixative (6-24 h), processed for paraffin embedding in vacuum and cut at a thickness of 5-7 $\mu \mathrm{m}$. The avidinbiotin-peroxidase complex $(A B C)$ method was performed with the Vectastain $A B C$ kit (Vector Laboratories, Burlingame,CA, USA). Sections from intact and castrated rats were treated in the same run. They were deparaffinized in xylene and hydrated in a graded solution of ethanol. After the quenching of endogenous peroxidase activity in water containing $0.3 \%$ hydrogen peroxide for $30 \mathrm{~min}$, nonspecific binding was blocked by treatment with $1.5 \%$ normal goat serum (Vector) in $0.01 \mathrm{M}$ PBS (phosphate-buffered saline; $\mathrm{pH}$ 7.2) for $30 \mathrm{~min}$. Polyclonal rabbit anti-NGF (sc-549; Santa Cruz Biotechnology, Santa Cruz, CA, USA), -BDNF (sc546; Santa Cruz Biotechnology, Santa Cruz, CA., USA), - TrkB (sc-12; Santa Cruz Biotechnology, Santa Cruz, CA, USA), -p75 NTR (N 3908; SigmaAldrich, St Louis, MO, USA), and -TrkA sera (sc118; Santa Cruz Biotechnology, Santa Cruz, CA., USA) were singularly applied to the sections at a dilution of 1:500 and each specimen was incubated in a moist chamber overnight at $4^{\circ} \mathrm{C}$. After the sections had been washed three times in PBS, biotinylated anti-rabbit IgG (Vector) was applied at a dilution of 1:200. The sections were again incubated for 30 min at room temperature. Freshly prepared $A B C$ reagent (Vector) was applied and incubated for 30 min after three washes in PBS. The localization of immunoreactions was visualized by incubating the sections for 5 min in freshly prepared diaminobenzidine-nickel solution (Vector).

The specificity of the immunoreactions was tested by replacing the primary antibody with buffer. No immunoreaction was detected in control tests. The specificity of the primary antibodies were tested by adsorption with excess (up to $50 \mu \mathrm{g} / \mathrm{mL}$ in the final dilution) homologous antigen peptide (NGF peptide sc-549P, BDNF peptide sc-546 P, TrkB peptide sc-118P, $p 75^{\text {NTR }}$ peptide sc-37268 and TrkA peptide sc-118P; Santa Cruz Biotechnology, CA, USA). 


\section{Imaging, quantification and statistical analysis}

Ten sections (one section every ten sections) for each ganglion of normal and castrated rats were independently evaluated by two observers by using a light microscope. Images were acquired digitally with a Nikon DS-LI camera. The images were analysed with the public domain NIH Image $\mathrm{J}$ software (http://rsb.info.nih.gov/nih-image/). Cell counting ( $\%$ positive cells/total number) and measurement of soma area $\left(\mu m^{2}\right)$ was performed on immunoreactive and negative ganglion neurons. The measurement of soma area was performed by manually outlining soma profiles on the computer screen. All data has been expressed as mean \pm SEM, where $n=$ number of animals. Two groups of data were compared (e.g. normal vs castrated) by using unpaired one-way Student's t-test. For this test $p<0.05$ was regarded as significant.

\section{Results}

\section{NGF-immunoreactivity}

NGF-IR was observed in the neurons of the pelvic ganglia of normal (Figure $1 \mathrm{la}$ ) and castrated rats (Figure $1 \mathrm{~b}$ ). About $80 \%$ of the total number of the counted cells was positive (Figure $3 a$ ). No statistically significant differences in the number of the immunoreactive neurons (Figure 3a).

\section{BDNF-immunoreactivity}

BDNF-IR was observed in the neurons of the MPG and accessory ganglia of normal (Figure 1c, d) and castrated rats (Figure le). In normal rats, about $80 \%$ of the total number of the counted cells were immunoreactive (Figure $3 a$ ). In castrated rats a significant decrease of the number of immunoreactive cells (Figure $3 a$ ) was observed.
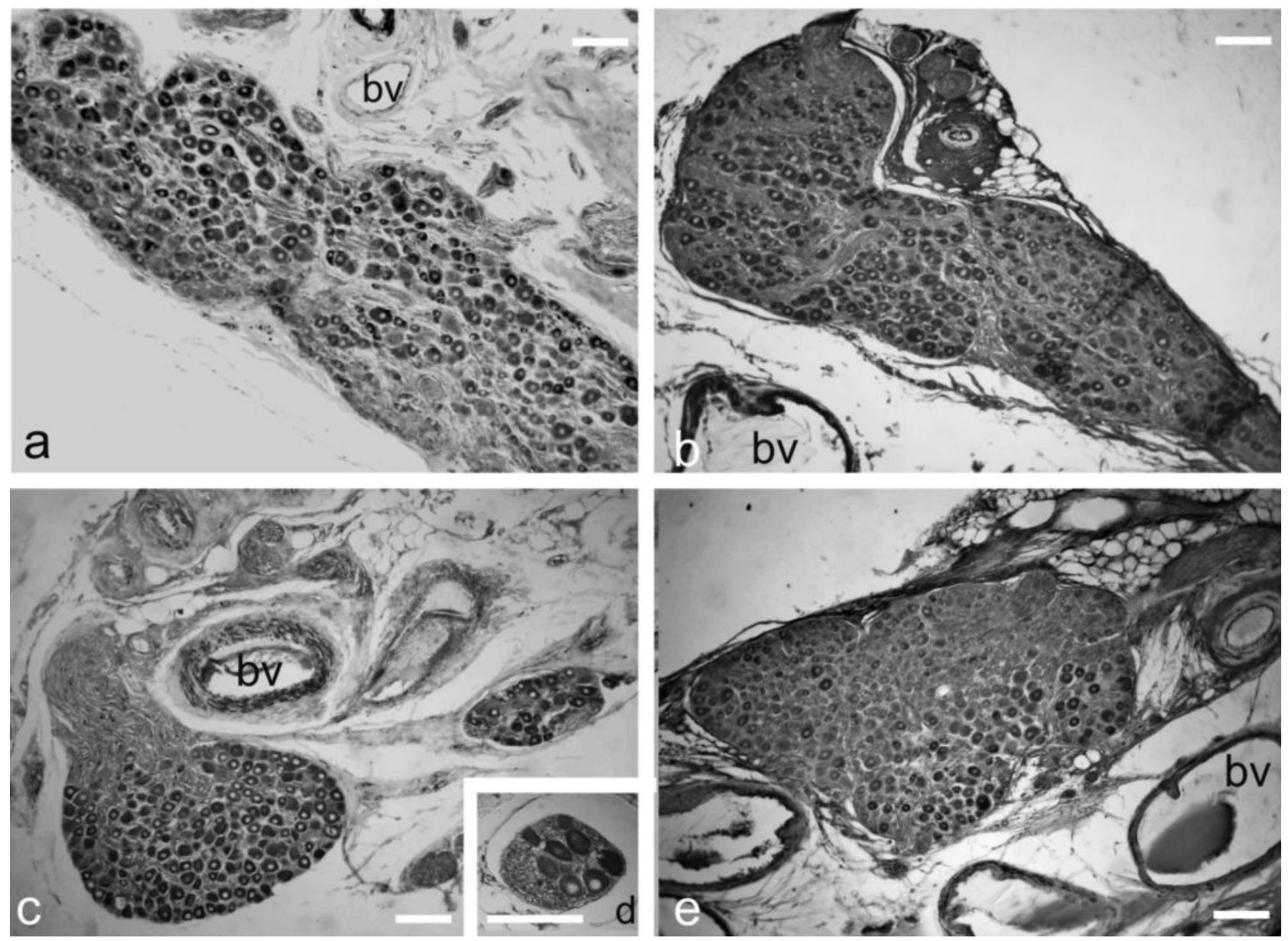

Figure 1. NGF- and BDNF-IR in the pelvic ganglia of the normal and castrated rats. NGF-IR was observed in MPG of the normal (a) and castrated (b) rats. BDNF-IR was observed in MPG (c, e) and in accessory ganglia (d) of the normal (c, d) and castrated (e ) rats. bv blood vessels. Bar $50 \mu \mathrm{m}$. 


\section{TrkB-immunoreactivity}

TrkB-IR was found in the neurons of the pelvic ganglia of normal (Figure 2a) and castrated rats (Figure 2b). In addition TrkB-IR was found in some preganglionic nerve fibres which contact pelvic neurons (data not shown). About $80 \%$ of the total number of the counted cells were immunoreactive. No significant differences was found in the number of TrkB-IR neurons after castration (Figure 3a).

\section{p75 ${ }^{\text {NTR }}$-immunoreactivity}

p75 ${ }^{\text {NTR }}$-IR was observed in the neurons of the pelvic ganglia of normal (Figure 2C) and castrated rats (Figure $2 d$ ). About $95 \%$ of the total number of the counted cells was immunoreactive (Figure 3a). A statistically significant reduction in the number of $\mathrm{p} 75^{\mathrm{NTR}}$-IR neurons were found after castration (Figure $3 a$ ).
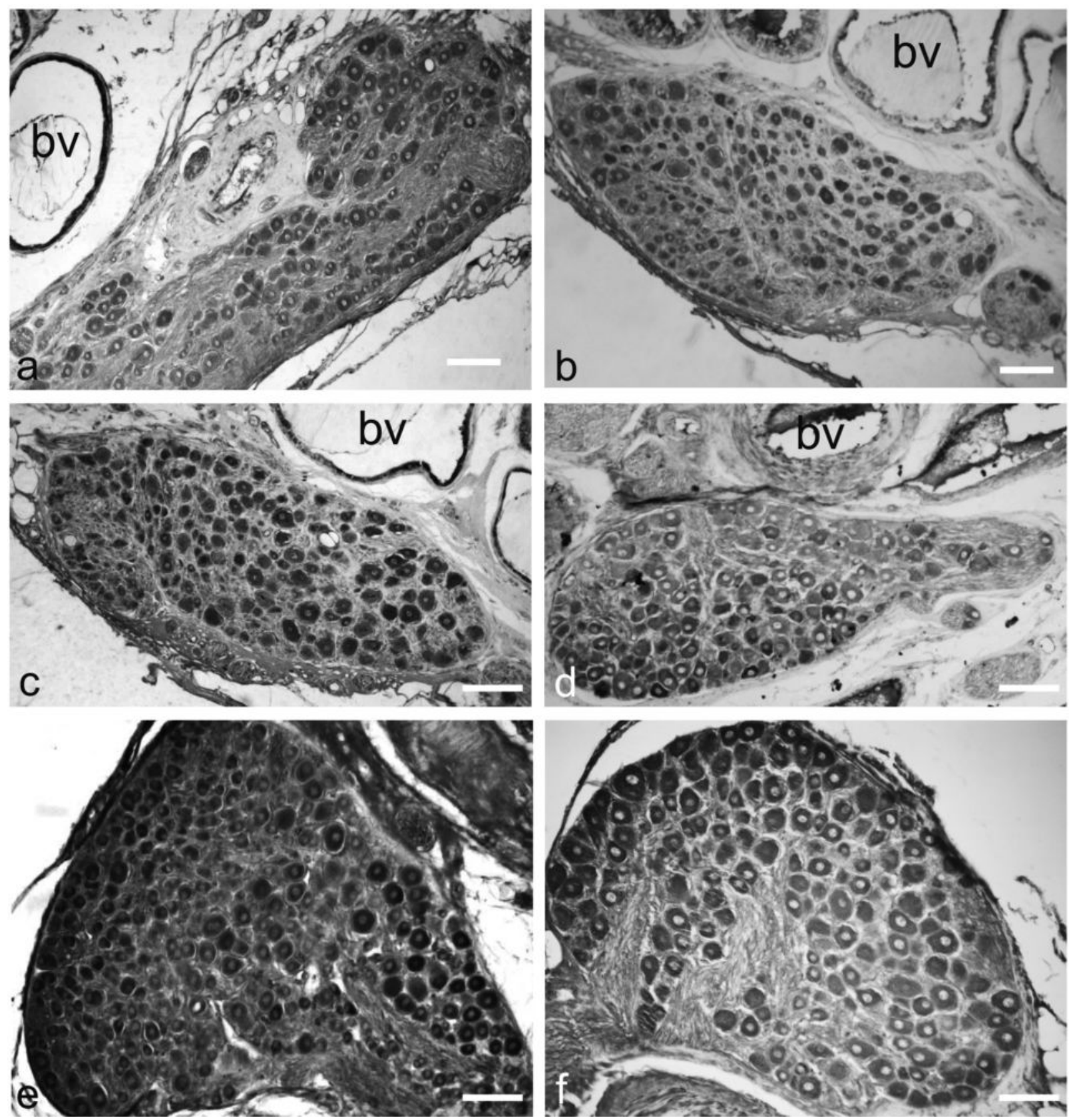

Figure 2. TrkB-, $p 75^{\text {NTR }}$ and TrkA-IR in the pelvic ganglia of the normal and castrated rats. TrkB-IR was observed in MPG of the normal (a) and castrated (b) rats. p75NTR-IR was observed in MPG of the normal (c) and castrated (d) rats. TrkA-IR was observed in MPG of the normal (e) and castrated (f) rats Bv blood vessels. Bar $50 \mu \mathrm{m}$. 


\section{TrkA-immunoreactivity}

TrkA-IR was found in neurons of the pelvic ganglia of normal (Figure 2e) and castrated rats (Figure $2 \mathrm{f}$ ). About $80 \%$ of the total number of the counted cells were immunoreactive (Figure $3 a$ ). No statistically significant differences in the number of immunoreactive neurons was observed after castration.

\section{Effects of castration on the soma size}

In castrated rats a significant decrease of the soma size of NGF-, BDNF-, TrkB-, p75 ${ }^{\mathrm{NT}_{-}}$and TrkA-IR cells (Figure 3b) was observed. A significant decrease of the soma size in negative neurons was also observed after castration (Figure $3 b$ ).
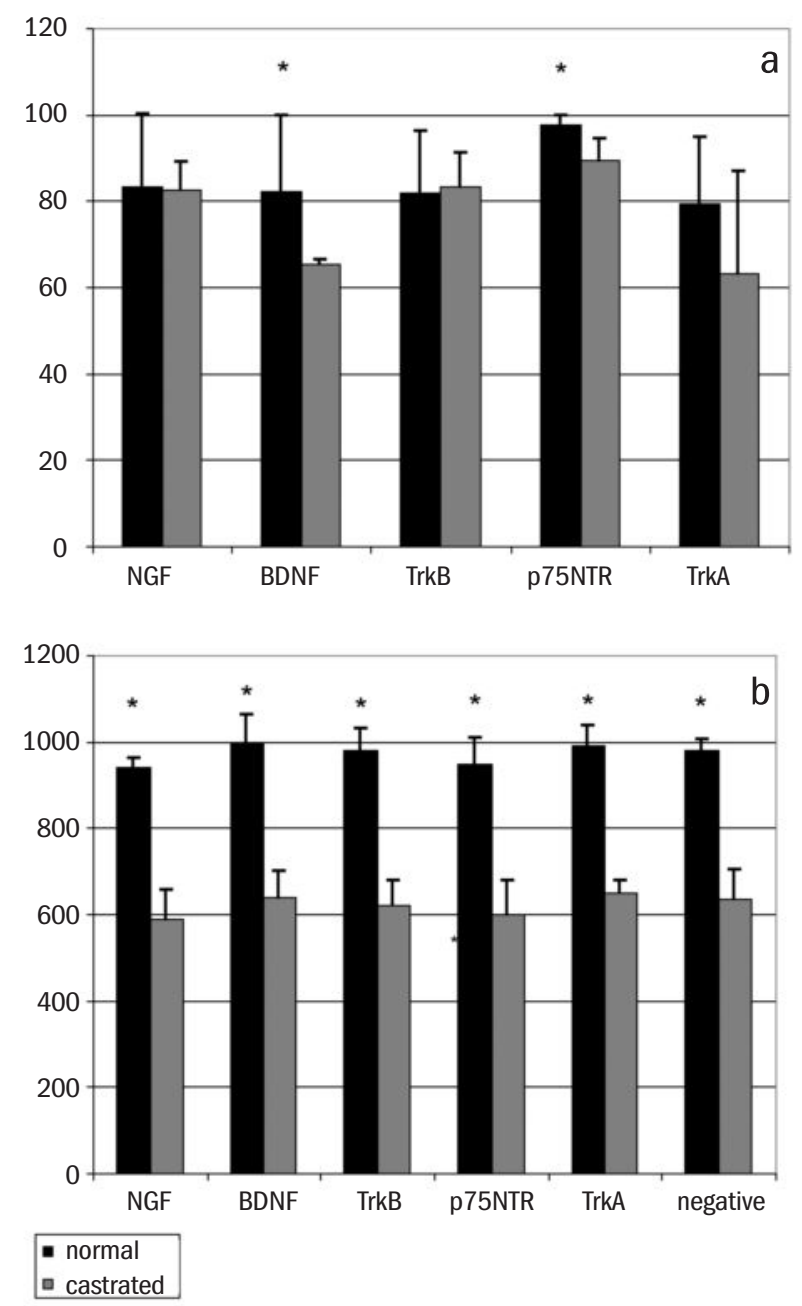

Figure 3. Effects of the castration on the number and soma area of the immunoreactive pelvic ganglia neurons. Histograms in the panel a show the percentage of immunoreactive cells on the total number in the pelvic ganglia of the normal and castrated rats. Histograms in the panel b show the soma area $\left(\mu \mathrm{m}^{2}\right)$ of the pelvic ganglion neurons in the normal and castrated rats. All data represent Mean \pm S.E.M. Animal number $=5$, ${ }^{\star} p<0,05$.

\section{Discussion}

The results of the present study demonstrate that neurons in the pelvic ganglia of normal and castrated male rats were immunoreactive to $N G F, B D N F$ and their receptors (TrkA, TrkB and $p 75^{\mathrm{NTR}}$ ). NGFand BDNF-IRs have been previously found by using ELISA in the MPG ganglia of male rats (Murray et al. 2004). Moreover, TrkA-, TrkB- and p75 ${ }^{\text {NTR }}$-IRs have been found by using immunohistochemistry in the majority of neurons of these ganglia (Keast and Kepper, 2001; Murray et al. 2004).

About $80 \%$ of the total number of the ganglion cells were immunoreactive to each of the studied neurotrophins as well as to each of their receptors, thus suggesting a coexpression of NGF, BDNF and their receptors in male rat pelvic neurons. It is well known that neurotrophins actually act as target derived neurotrophic factors. However, the presence of both neurotrophins and their receptors in these neurons is also consistent with the hypothesis that neurotrophins could act through an autocrine/ paracrine mechanism in influencing pelvic neuronal functions. This hypothesis is supported by the fact that autonomic neurons are capable of synthesize both neurotrophins and their receptors (Hasan et al., 2003; Hiltunen et al., 2005). Immunoreactivity to neurotrophins and their receptors persisted after castration thus suggesting a role for neurotrophins in regulating the castration-induced involutive processes occurring in pelvic neurons. However, a significant decrease in the number of BDNF-IR neurons was observed in castrated rats. This result suggests that androgens positively modulate BDNF synthesis and/or its receptor affinity in the pelvic neurons. This seems to be in contrast with previous findings reporting an increase of BDNF expression in the accessory male genital glands (i.e. one of the targets of the pelvic innervation) of the rat after castration (Mirabella et al., 2006; Mirabella et al., 2007). Thus, a difference can be hypothesized for androgen hormones in influencing their target tissues.

TrkB-immunoreactivity was found in the pelvic neurons of the normal and castrated rats. After castration, no difference in the number of immunoreactive neurons was observed. This appears in contrast with the previous finding reporting a decrease in the number of BDNF-IR neurons. The reasons of this discrepancy remain to 
be investigated. However, the possibility that, the involutive processes occurring after castration are mediated by NT4/5, the other specific ligand of TrkB cannot be excluded. It has been observed that NT4/5 by binding TrkB influences pelvic ganglion neurons ( $L$ in et al., 2003).

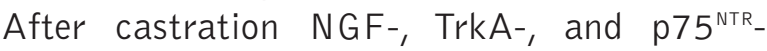
immunoreactivities were still present in the pelvic neurons. No statistically significant differences were observed in the number of NGF- and TrkAimmunoreactive neurons after castration. On the contrary, a statistically significant reduction in the number of $\mathrm{p} 75^{\mathrm{NTR}}$-immunoreactive neurons was observed in the castrated rats.

The data regarding $p 75^{\mathrm{NTR}}$-immunoreactivity appear in contrast with previous findings reporting an increase of $p 75^{\mathrm{NTR}}$-immunoreactivity in the nerves of the internal genital organs of castrated rats (Mirabella et al., 2007). The reasons of this discrepancy remain to be clarified. A significant decrease of the soma size of both immunoreactive and negative neurons was observed after castration. The degree of the reduction of the soma size was same regardless of the immunoreactivity to neurotrophin and their receptors. These results agree with previous findings demonstrating that pelvic neurons undergo a marked reduction in soma size under conditions of androgen deficiency (Keast and Saunders, 1998; Keast, 2000; Keast et al. 2002).

In conclusion, the results of the present study have shown that immunoreactivity to neurotrophin (i.e. NGF, BDNF) and their receptors (i.e. TrkA, Trk $B, p 75^{\text {NTR }}$ ) is expressed in the pelvic ganglia of normal and castrated rats. The immunohistochemical expression of NGF, BDNF and their receptors seems therefore differently regulated by androgens hormones.

\section{References}

Barbacid M. Neurotrophic factors and their receptors. Curr Opin Cell Biol 1995; 7: 148-55.

Chao MV. Neurotrophins and their receptors: A convergence point for many signaling pathways. Nat Rev 2003;4:299-308.

Ernfors P, Lee KF, Kucera J, Jaenisch R. Lack of neurotrophin-3 leads to deficiencies in the peripheral nervous system and loss of limb proprioceptive afferents. Cell 1994;77: 503-12.

Hasan W, Pedchenko T, Krizsan-Agbas D, Baum L, Smith PG. Sympathetic neurons synthesize and secrete pro-nerve growth factor protein. J Neurobiol 2003;57:38-53.

Hiltunen J0, Laurikainen A, Klingeb E, Saarmaa M . Neurotrophin-3 is a target-derived eurotrophic factor for penile erection-inducing neurons. Neuroscience 2005;133:51-8.
Huang EJ, Reichart LF. Neurotrophins: roles in neuronal development and function. Annu Rev Neurosci 2001;24:677-736.

Keast JR. Effects of testosterone on pelvic autonomic pathways: progress and pitfalls. J Auton Nerv Syst 2000;79:67-73.

Keast JR. Plasticity of pelvic autonomic ganglia and urogenital innervation. Int Rev Cytol 2006;248:141-208.

Keast JR, Gleeson RJ, Shulkes A, Morris MJ. Maturational and maintenance effects of testosterone on terminal axon density and neuropeptide expression in the rat vas deferens. Neuroscience 2002; 112:391-8.

Keast JR, Kepper ME. Differential regulation of TrkA and p75NTR in noradrenergic pelvic autonomic ganglion cells after deafferentation of their cholinergic neighbours. Eur J Neurosci 2001;13:211-20.

Keast JR, Saunders RJ. Testosterone has potent, selective effects on the morphology of pelvic autonomic neurons which control the bladder, lower bowel and internal reproductive organs of the male rat. Neurosci 1998; 85: 543-56.

Leon A, Buriani A, Dal Toso R, Fabris M, Romanello S, Aloe L, LeviMontalcini R. Mast cells synthesize, store, and release nerve growth factor. Proc Natl Acad Sci USA 1994;91: 3739-43.

Levi-Montalcini R. The nerve growth factor 35 years later. Science 1987;237:1154-62.

Lewin GR, Barde YA. Physiology of neurotrophins. Annu Rev Neurosci 1996;19:289-317.

Lin G, Chen KC, Hsieh PS, Yeh CH, Lue TF, Lin CS. Neurotrophic effects of vascular endothelial growth factor and neurotrophins on cultured major pelvic ganglia. BJU Int 2003;92:631-5.

Mirabella N, Squillacioti C, Varricchio E, Genovese A, Paino G. Innervation of vas deferens and accessory male genital glands in the water buffalo (Bubalus bubalis). Neurochemical characteristics and relationships to the reproductive activity. Theriogenology 2003;59: 1999-2016.

Mirabella N, Squillacioti C, Paone I, Ciarcia R, Russo M, Paino G. Effects of castration on the expression of brain-derived neurotrophic factor (BDNF) in the vas deferens and male accessory genital glands of the rat. Cell Tissue Res 2007;323:513-22.

Mirabella N, Squillacioti C, De Luca A, Paino G. Effects of castration on the expression of TrkB and p75NTR in the vas deferens and male accessory genital glands of the rat. Anat, Histol, Embryol 2008; doi:10.1111/j.1439-0264.2007.00804.x.

Murer MG, Yan Q, Raisman-Vozari R. Brain-derived neurotrophic factor in the control human brain, and in Alzheimer's disease and Parkinson's disease. Prog Neurobiol 2001;63:71-124.

Murray E, Malley SE, Qiao LY, Hu VY, Vizzard MA. Cyclophosphamide induced cystitis alters neurotrophin and receptor tyrosine kinase expression in pelvic ganglia and bladder. J Urol 2004;172:2434-9.

Patapoutian A, Reichart LF. Trk receptors: mediators of neurotrophin action. Curr Opin Neurobiol 2001;11:272-80.

Purinton PT, Fletcher, Radley WE. Gross and light microscopic features of the pelvic plexus in the rat. Anat Rec 1973;175:697-706.

Rosenbaum T, Vidaltamayo R, Sanchez-Soto MC, Zentella A, Hiriart M. Pancreatic beta cells synthesize and secrete nerve growth factor. Proc Natl Acad Sci USA 1998;95:7784-8.

Sariola $\mathrm{H}$. The neurotrophic factors in non-neuronal tissues. Cell Mol Life Sci 2001;58:1061-6.

Schinder AF, Poo M. The neurotrophin hypothesis for synaptic plasticity. Trends Neurosci 2000;23:639-45.

Snider WD. Function of the neurotrophins during nervous system development: what the knockouts are teaching us. Cell 1994;77:627-38.

Teng KK, Hempstead BL. Neurotrophins and their receptors: Signaling trios in complex biological systems. Cell Mol Life Sci 2004; 61:3548.

Tessarollo L. Pleiotropic functions of neurotrophins in development. Cytokine Growth Factor Rev 1998;9:125-37.

Yamamoto M, Sobue G, Yamamoto K, Terao S. Expression of mRNAs for neurotrophic factors (NGF, BDNF, NT-3 and GDNF) and their receptors ( $p 75 N G F$, trkA, trkB and trkC) in the adult human peripheral nervous system and nonneural tissues. Neurochem Res 1996; 21:929-38. 